\title{
MPMI ACKNOWLEDGMENT OF REVIEWERS, 2021
}

The success of Molecular Plant-Microbe Interactions depends on the quality of manuscripts submitted by authors and on the care and competence with which they are reviewed. It is the policy of the Editorial Board to solicit reviews of manuscripts from specialists most qualified to review them. In addition to members of the Editorial Board, the individuals listed below provided constructive critical reviews of one or more manuscripts during the past year. Their names are published here in grateful appreciation for their contributions to the journal.

Pervaiz Abbasi

Reem Aboukhaddour

Paul Abraham

Gerhard Adam

Gladys Alexandre

Jeff Anderson

Jeffrey Anderson

Maxuel Andrade

Jean-Michel Ané

Nathalie Aoun

Klaus-J. Appenroth

Chris Argueso

Federico Ariel

Andrew Armitage

Kyaw Aung

Dilay Hazal Ayhan

Ofir Bahar

Gerd Balcke

David Baltrus

Mark Banfield

Riccardo Baroncelli

Laura Baxter

Daniel Bebber

Faith Belanger

Richard Bélanger

Gilles Bena

Celso Benedetti

Ximena Besoain

Ton Bisseling

Alex Blacutt

Anna Block

Ana Maria Bocsanczy

Melvin Bolton

Orlando

Borrás-Hidalgo

Marc Bramkamp

Mathias Brands

Armando Bravo

Eric Brenya

Marin Brewer

Martin Broberg

Daren Brown

Damian Cambiagno

Delphine Capela

Jeffrey Caplan

Morgan Carter

Sergio Casas-Flores

Christian Danve

M. Castroverde

Eunyoung Chae

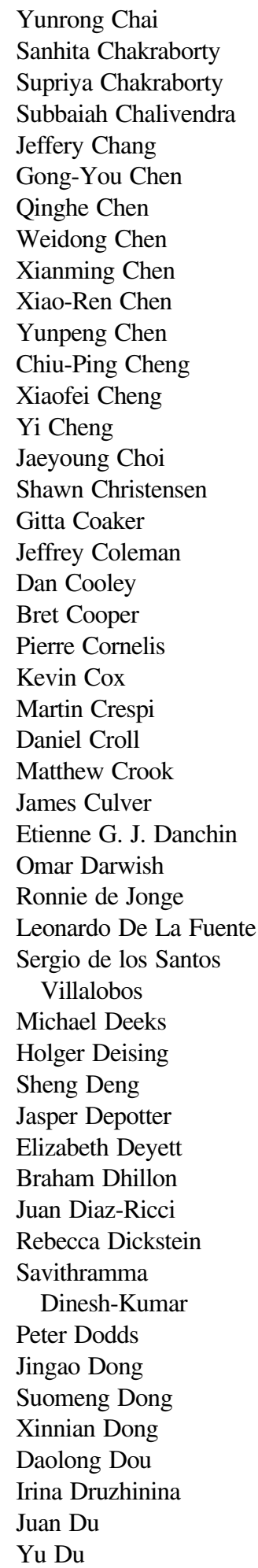

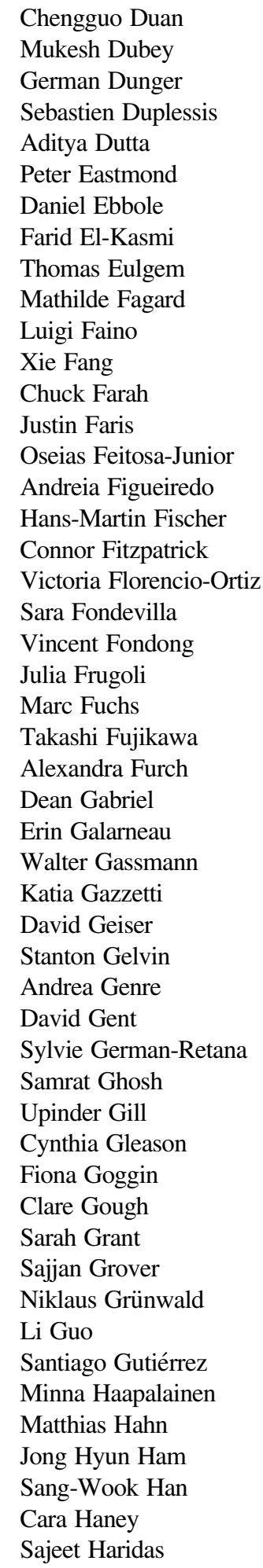

Gary Harman

Maria Harrison

Anton Hartmann

Baoye He

Sheng-Yang He

Xinyao He

Ya-Wen $\mathrm{He}$

Jason Heindl

Tyler Helmann

Adriana Hemerly

Holger Heuer

Thierry Heulin

Benjamin Horwitz

Chuan-Chih Hsu

Xiaoping $\mathrm{Hu}$

Jian Hua

Shuai Huang

Wei Huang

Chen huilan

Giuseppe Ianiri

Michael Ionescu

Stephanie Jaubert

Jay Jayaraman

Junhyun Jeon

Daohong Jiang

Kenneth Johnson

Jonathan Jones

Matthias Jost

Jennifer Jungfleisch

Yasuhiro Kadota

Kenji Kai

Peter Kalo

Petr Karlovsky

Takao Kasuga

Shinpei Katou

Masayoshi Kawaguchi

Beat Keller

Kyoung-Su Kim

Min-Sung Kim

Saet-Byul Kim

Sun Tae Kim

Ralph Kissen

H. Corby Kistler

Ralf Koebnik

Ksenia Krasileva

Christian P. Kubicek

Yasuyuki Kubo

Aditya Kulshreshtha

Stefan Kusch

Brian Kvitko 
Tina Kyndt

Eric Lam

Sarah Lebeis

Julien levy

Bo Li

Yaqian Li

Yan Liang

Judith Lichtenzveig

Hsiao-Han Lin

Nai-Chun Lin

Bao Liu

Huiquan Liu

Sanzhen Liu

Wede Liu

Yule Liu

Francisco Lopez-Baena

Emilia Lopez-Solanilla

Zhao-Qing Luo

Zhonghua Ma

Alberto Macho

April MacIntyre

Kristiina Mäkinen

Mathu Malar C

Robert Malinowski

Kranthi Mandadi

Ramona Marasco

Gregory Martin

German Martinez

Nani Maryani

Honour McCann

John McDowell

Jamie McGowan

Lauren McKee

Timothy McNellis

Paul Melloy

Kristin Mercer

Johana Misas Villamil

Antonio Molina

K. K. Mondal

Tomohiro Morohosi

Matthew Moscou

Glora Mosquera

G. Adam Mott

Zhonglin Mou

Gary Muehlbauer

Takafumi Mukaihara

Jesus Murillo

Jeremy Murray

Hirofumi Nakagami

Vamsi Nalam

Jason $\mathrm{Ng}$

Tatsuya Nobori

Yoshiteru Noutoshi
Thorsten Nuernberger

Richard O'Connell

Chang-Sik Oh

Giles Oldroyd

Ebrahim Osdaghi

Ralph Panstruga

Jane Parker

Katarzyna Parys

Uta Paszkowski

Hitendra Patel

Julia Pawłowska

Katharina Pawlowski

Yann Pecrix

Set Perez

Elsa Petit

Catalina Pislariu

Peter Poor

Neha Potnis

Humberto Prieto

Guo-Liang Qian

Wei Qian

Yongli Qiao

Michael Quentin

Ashish Ranjan

Ravi Ranjan

Pascal Ratet

Brandon Reagan

Gabriel Rech

Didier Reinhardt

Martijn Rep

Thomas Rey

Philippe Reymond

Jonathan Richards

Christopher Ridout

Bernardo Rodamilans

Alejandro Rojas

Jeffrey Rollins

Lina Russ

Yusuke Saijo

Pasquale Saldarelli

Rozalynne Samira

Siva Sankaran

Aditya Sarangi

Panagiotis Sarris

Tim Satterlee

Diane Saunders

Michael Savka

Patrick Schäfer

Birgit Scharf

David Scheuring

Barbara Schulz

Steve Scofield

Cecile Segonzac
Seungyeon Seo

Young-Su Seo

Hope Serepa-Dlamini

Libo Shan

Itai Sharon

Danyu Shen

Zedan Shen

Ken Shirasu

Karam Singh

Alok Sinha

Geert Smant

Junqi Song

Edgar Spalding

Jana Sperschneider

Francesco Spinelli

John Stavrinides

Adam Steinbrenner

Wolfgang Stöggl

Ioannis Stringlis

Rajagopal

Subramaniam

Senthil Subramanian

Masayuki Sugawara

Nobuhiro Suzuki

Bryan Swingle

Javier Tabima

James Tambong

Kar-Chun Tan

Kiwamu Tanaka

Matthew Tancos

Wei-Hua Tang

Stefania Tegli

Jorge Teodoro de Souza

Ryohei Terauchi

Arinthip

Thamchaipenet

Shree Thapa

Bart Thomma

David Thoms

Chang Fu Tian

Jens Tilsner

Sujan Timilsina

Christian Tobias

Peri Tobias

Yukio Tosa

Anil Tripathi

Lisa Vaillancourt

Marc Valls

Guido Van den

Ackerveken

Frederique Van

Gijsegem
Jan van Kan

Ramesh Vetukuri

Paulo Vieira

Jose Vinardell

Frank Waller

Bo Wang

Nian Wang

Qinhu Wang

Xuan Wang

Yiming Wang

Zonghua Wang

Hai-Lei Wei

Detlef Weigel

Frank White

Steven Whitham

Simon Williams

Joe Win

Kathrin Wippel

Lennart Wirthmueller

Thomas Wolpert

Brande Wulff

Nathan Wyatt

Haiping Xin

Shuichi Yanagisawa

Fenghuan Yang

Hong Yang

Wannian Yang

Xingyong Yang

Yu-Liang Yang

Nan Yao

Wenwu Ye

Chuntao Yin

Heejin Yoo

Hirofumi Yoshioka

Houlin Yu

Manda Yu

Lirong Zeng

Haifeng Zhang

Jianping Zhang

Meixiang Zhang

Xiong Zhang

Yong Zhang

Yuqin Zhang

Bingyu Zhao

Yi Zhao

Youfu "Frank" Zhao

Lu Zheng

Jie Zhong

Jianmin Zhou

Hongyan Zhu 